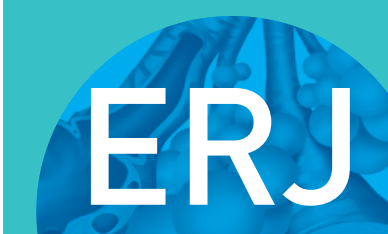

open research

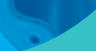

\title{
Pleuroparenchymal fibroelastosis in Mycobacterium avium complex pulmonary disease: clinical characteristics and prognostic impact
}

\author{
Yuji Yamamoto (1) ${ }^{1,2}$, Kazuyuki Tsujino', Tomoki Kuge ${ }^{1}$, Fukuko Okabe², \\ Takahiro Kawasaki ${ }^{1}$, Takanori Matsuki ${ }^{1}$, Hiroyuki Kagawa ${ }^{1}$, Mari Miki ${ }^{1}$, \\ Keisuke Miki ${ }^{1}$, Masahide Mori ${ }^{2}$ and Hiroshi Kida (ib ${ }^{1}$
}

Affiliations: 'Dept of Respiratory Medicine, National Hospital Organization Osaka Toneyama Medical Center, Toyonaka, Japan. ${ }^{2}$ Dept of Thoracic Oncology, National Hospital Organization Osaka Toneyama Medical Center, Toyonaka, Japan.

Correspondence: Yuji Yamamoto, Dept of Respiratory Medicine, National Hospital Organization Osaka Toneyama Medical Center, 5-1-1 Toneyama, Toyonaka, Osaka 560-8552, Japan.

E-mail: cyyamamoto1110agmail.com

ABSTRACT The association between Mycobacterium avium complex pulmonary disease (MAC-PD) and pleuroparenchymal fibroelastosis (PPFE) has been reported previously, and interstitial pneumonia as a comorbidity is associated with a worse prognosis. However, no study has thoroughly reported on PPFE associated with MAC-PD. The present study investigated the prevalence, clinical characteristics, and prognostic impact of PPFE in patients with MAC-PD.

A total of 224 patients, newly diagnosed with MAC-PD, were retrospectively reviewed. At the time of diagnosis, chest high-resolution computed tomography (HRCT), sputum examination, and clinical characteristics were collected. The extent of PPFE and MAC-PD was evaluated semi-quantitatively using HRCT scores. Risk factor analysis for clinical or radiological deterioration necessitating multidrug antimicrobial treatment within 3 years, and all-cause mortality within 5 years, from the initial diagnosis was performed based on the PPFE score.

PPFE was observed in 59 out of 224 patients (26.3\%). A higher PPFE score was a risk factor for dyspnoea, fatigue, and lower body mass index (BMI) $(\mathrm{p}<0.05)$. Although PPFE score did not correlate with clinical or radiological deterioration within 3 years $(\mathrm{p}=0.576)$, a higher PPFE score (adjusted OR 1.66, 95\% CI 1.06-2.60, p=0.028) and lower BMI (adjusted OR 0.61, 95\% CI 0.39-0.94, $\mathrm{p}=0.028$ ) increased the risk of 5 -year mortality.

PPFE is a relatively common complication and an independent poor prognostic factor of MAC-PD. This study highlights the need for further studies investigating whether the presence of PPFE can be a clinical indicator for initiating treatment of MAC-PD.

@ERSpublications

The presence of pleuroparenchymal fibroelastosis in patients with Mycobacterium avium complex pulmonary disease is a risk factor for lower body mass index and worse prognosis https://bit.ly/3g8D6Di

Cite this article as: Yamamoto Y, Tsujino K, Kuge T, et al. Pleuroparenchymal fibroelastosis in Mycobacterium avium complex pulmonary disease: clinical characteristics and prognostic impact. ERJ Open Res 2021; 7: 00765-2020 [https://doi.org/10.1183/23120541.00765-2020].

This article has supplementary material available from openres.ersjournals.com.

Received: 20 Oct 2020 | Accepted: 29 Nov 2020

Copyright $\odot$ ERS 2021. This article is open access and distributed under the terms of the Creative Commons Attribution Non-Commercial Licence 4.0. 


\section{Introduction}

Non-tuberculous mycobacterial (NTM) pulmonary disease (PD) has been increasingly implicated in a broad range of infectious diseases worldwide $[1,2]$. Mycobacterium avium complex (MAC), predominantly comprising M. avium and M. intracellulare, is the most common aetiology of NTM-PD [3]. MAC-PD has two major clinical phenotypes: fibrocavitary (FC) and nodular bronchiectatic (NB) phenotypes [4]. Patients with the FC phenotype have a worse prognosis than those with the NB phenotype $[5,6]$, and even the NB phenotype is a risk factor for re-infection and relapse [3]. In addition to these two radiological characteristics, patients with MAC-PD occasionally present with pleuroparenchymal fibroelastosis (PPFE) [7].

PPFE is a specific clinical-pathological entity that AMITANi et al. [8] and Frankel et al. [9] first reported. Its pathogenesis remains unclear, but earlier studies reported that PPFE was associated with a variety of diseases, including autoimmune diseases, aspergillosis and NTM infection [7, 10-13]. Moreover, patients with PPFE and granulomatous diseases, including aspergillosis and MAC infection, had a better prognosis than those with PPFE without granulomas [7]. However, the association between PPFE and MAC-PD has not been thoroughly investigated. In particular, no study has evaluated the prevalence, clinical and radiological characteristics, and prognostic impact of PPFE in patients with MAC-PD. As the presence of PPFE indicates a poor prognosis in other diseases $[11,13]$, we hypothesised that PPFE might induce severe clinical symptoms and be associated with a poor prognosis in patients with MAC-PD.

The aims of this study were to estimate the prevalence of radiological PPFE in patients with MAC-PD, to assess the clinical characteristics and the prognostic impact of PPFE, and to evaluate the correlation between radiological PPFE and the radiological and clinical features of MAC-PD.

\section{Methods}

Patients and study design

This retrospective study was performed in accordance with the Declaration of Helsinki. The Institutional Review Board of the National Hospital Organisation (NHO) Osaka Toneyama Medical Center approved the study protocols and chose an opt-out system for obtaining patients' informed consent (approval number: TNH-P-2020043). All adult patients with MAC-PD who attended clinics at the NHO Osaka Toneyama Medical Center between January 2012 and December 2016 were screened. Patients with a previous history of antimicrobial treatment for MAC-PD were excluded, as well as those who did not meet the diagnostic criteria of MAC-PD [14]. Patients who did not undergo high-resolution computed tomography (HRCT) were also excluded. A patient inclusion flowchart is shown in figure 1. All patients

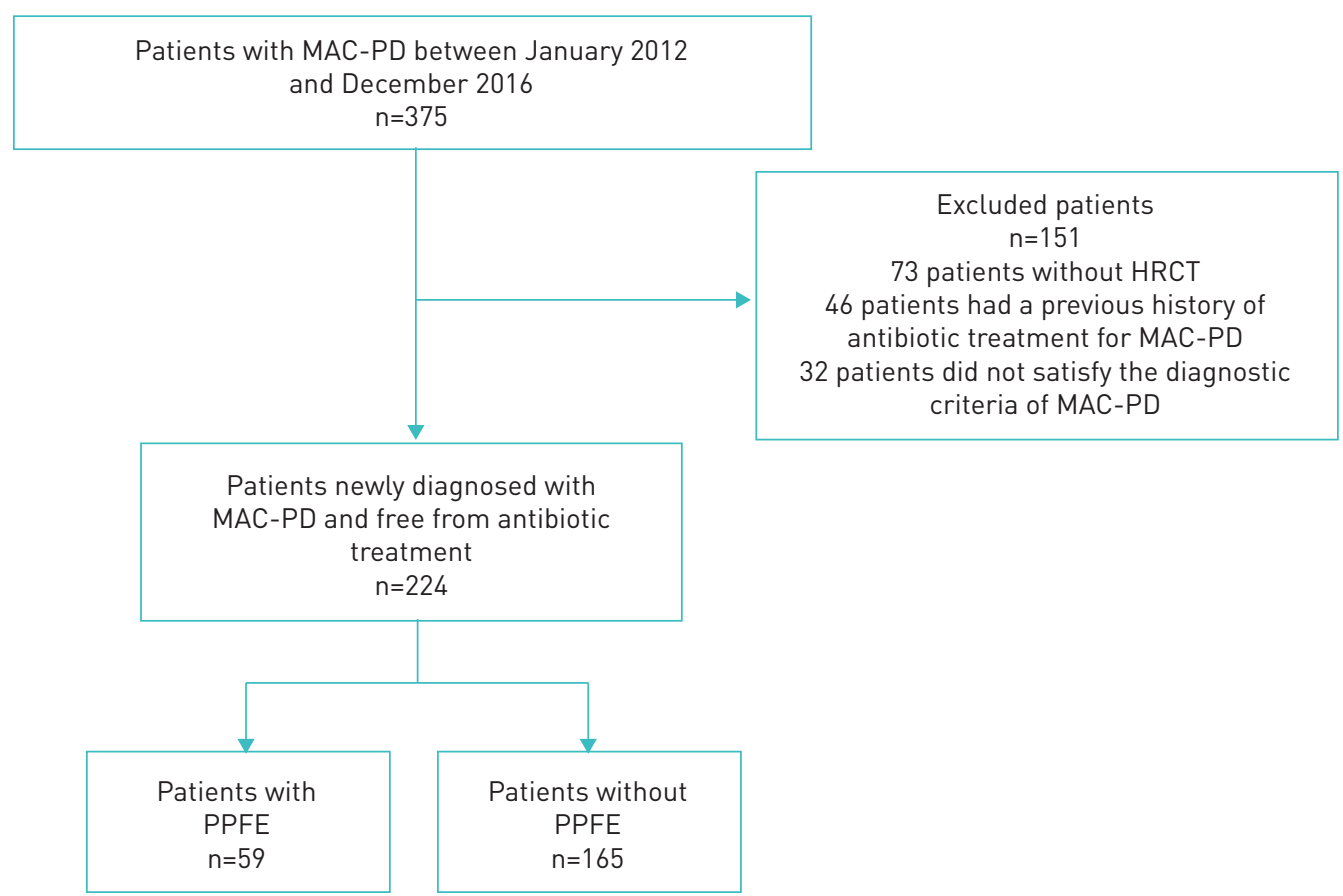

FIGURE 1 Inclusion flowchart. HRCT: high-resolution computed tomography; MAC-PD: Mycobacterium avium complex pulmonary disease; PPFE: pleuroparenchymal fibroelastosis. 
were followed until death, the end of the study period (September 30, 2020), or the end of the observational period, 5 years following initial diagnosis. Chest HRCT scans were followed at least once in a year and at the time of treatment initiation. Only patients who qualified for the study were evaluated.

\section{Data collection}

Clinical and radiological characteristics were collected by individual case review. Baseline data were obtained at the time of the initial diagnosis of MAC-PD. Clinical characteristics included age, sex, body mass index (BMI), and smoking status. Comorbidities included a previous history of pulmonary tuberculosis. Clinical symptoms such as cough, sputum, dyspnoea, fever, fatigue, and weight loss were noted. Acid-fast bacilli (AFB) smear, a history of multidrug antimicrobial treatment for MAC-PD within 3 years of diagnosis, and all-cause mortality within 5 years from the initial diagnosis were also recorded. Progressive disease was defined as requiring multidrug antimicrobial treatment within 3 years of the initial diagnosis due to clinical or radiological deterioration [5]. Stationary disease was defined as a patient free from multidrug antimicrobial treatment for at least for 3 years from the initial diagnosis without clinical or radiological deterioration. Radiological characteristics were evaluated using PPFE and MAC-PD scores on HRCT.

\section{Radiological PPFE diagnosis and HRCT score}

Chest HRCT scans were conducted with $1-\mathrm{mm}$ section thickness and evaluated semi-quantitatively by two pulmonologists trained in computed tomography scoring. A third scorer adjudicated discordant scores. Scorers assessed PPFE features and extent [10], as well as MAC-PD scores, without patients' clinical information. The extent of pleural surface involvement from PPFE in each lobe was evaluated on a four-point categorical scale: 0 : absent; 1 : mild, only affecting $<10 \%$ of the pleural surface; 2 : moderate, affecting $10-33 \%$ of the pleural surface; and 3: severe, affecting $>33 \%$ of the pleural surface [15]. Total PPFE scores ranged from 0 to 18 , with a score between 0 and 2 indicating limited disease, and $\geqslant 3$ indicating extensive disease. If the PPFE score was 1 or 2 , and pleural surface involvement was restricted to the uppermost $5 \mathrm{~mm}$ of each hemithorax, patients were not diagnosed with PPFE but with apical pleural caps (figure 2) [16, 17].

\section{MAC-PD HRCT scores}

MAC-PD severity was evaluated in each lobe on HRCT by scoring bronchiectasis (0-9 points), cellular bronchiolitis (0-6 points), cavity (0-9 points), nodules (0-3 points), and consolidation (0-3 points) (supplementary table 1) [18, 19]. The sum of the lobar scores for each HRCT finding were used in the analysis. Total MAC-PD scores ranged from 0 to 30.

\section{Sputum examination}

Expectorated sputum or bronchoscopic samples were examined using Ziehl-Neelsen staining. The results of smear microscopy were assessed semi-quantitatively. A positive smear was defined as one with $>1 \mathrm{AFB}$ per 100 high-power fields [20]. Sputum cultures were examined for AFB using 2\% Ogawa egg medium (Japan BCG, Tokyo, Japan) or a mycobacteria growth indicator tube (Japan Becton, Dickinson and Co., Tokyo Japan). NTM species were identified using the AccuProbe (Gen-Probe Inc., San Diego, CA, USA) or COBAS AMPLICOR (Roche Diagnostic, Tokyo, Japan) systems or by DNA-DNA hybridisation assay (Kyokuto Pharmaceutical Industrial, Tokyo, Japan).

\section{Statistical analysis}

All statistical analyses were performed using EZR version 1.38 (based on $\mathrm{R}$ version 3.5 .2 and $\mathrm{R}$ commander version 2.5-1; Jichi Medical University Saitama Medical Center, Saitama, Japan) [21]. Fisher's exact test and a t-test were used to compare characteristics between patients with and without radiological PPFE. Univariate and multivariate regression analyses were used for a correlation analysis between patient characteristics and PPFE score. Potential independent factors identified as significant by univariate analysis were evaluated using multivariate analysis, as well as age, sex, and BMI. For all analyses, a p-value $<0.05$ was considered statistically significant.

\section{Results}

\section{Patient characteristics}

A total of 224 patients with MAC-PD were identified and included in this study. The patients' baseline characteristics are shown in table 1 . The median age (interquartile range) was 68 (62-74) years. The patients were categorised radiologically into NB type $(n=173)$ and FC type $(n=48)$. Among the 103 patients with progressive MAC-PD, 86 received rifampicin (RFP)+ethambutol (EB)+clarithromycin (CAM)-based treatment regimens. RFP (300-450 mg), EB (500-750 mg), and CAM (600-800 mg) were prescribed daily. Aminoglycoside was injected in 43 patients (supplementary table 2). 

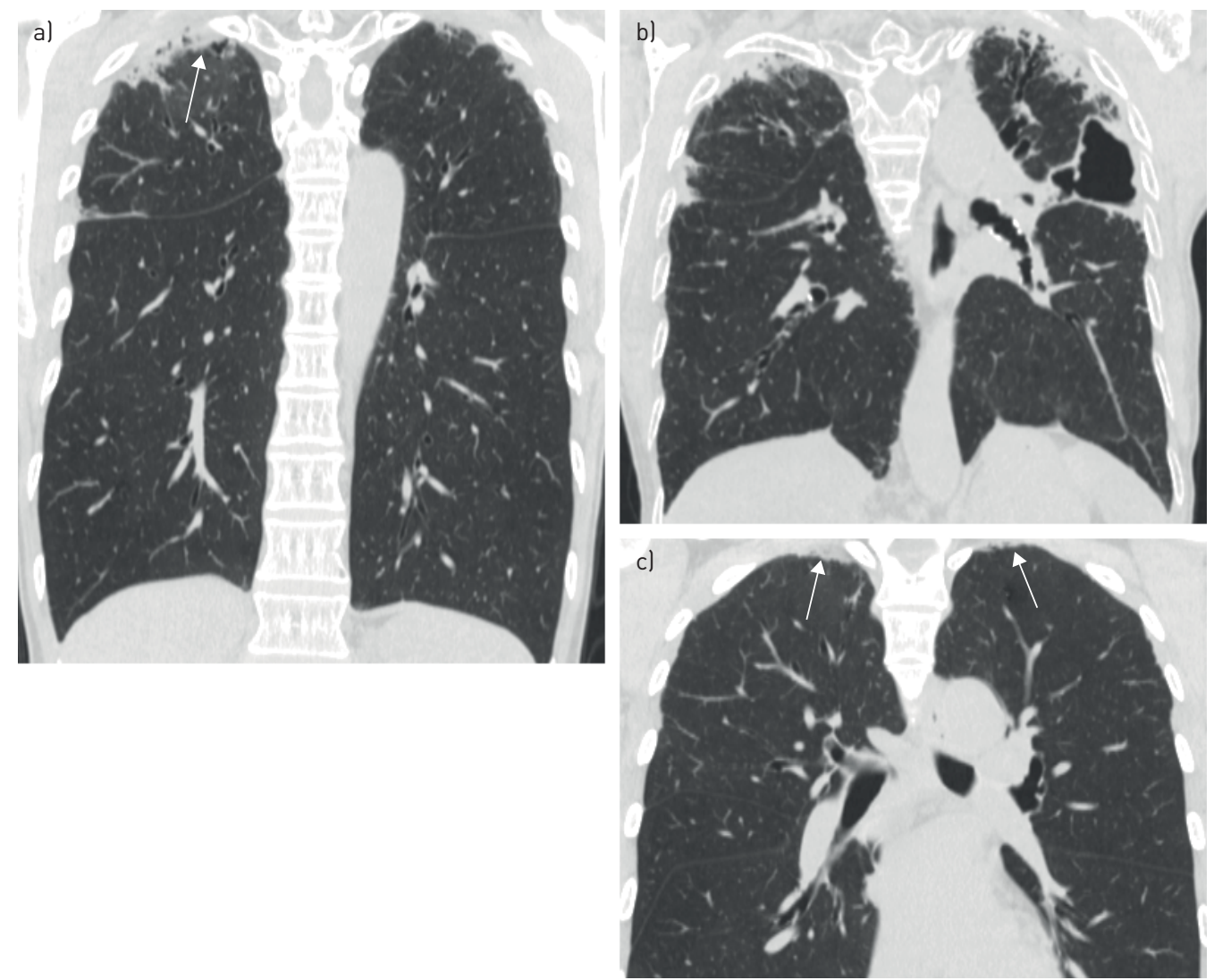

FIGURE 2 Radiological findings of pleuroparenchymal fibroelastosis (PPFE) and apical pleural caps in Mycobacterium avium complex pulmonary disease (MAC-PD). a) High-resolution computed tomography (HRCT) findings demonstrating typical features of PPFE. Pleural thickening, subpleural consolidation, and bronchiectasis/traction bronchiectasis can be observed (arrow). b) PPFE with cavitary lesions on the left upper lobe, indicating that PPFE and other radiological characteristics of MAC-PD can coexist in the lungs. c) HRCT findings of apical pleural caps (arrows).

Among the 88 patients with a positive PPFE score, 59 were diagnosed with PPFE, and the other 29 were diagnosed with apical pleural caps. Patients with PPFE were more likely to have a lower BMI, and greater age, smoking history, incidence of fatigue and weight loss, total MAC-PD score, and all-cause mortality within 5 years $(\mathrm{p}<0.05)$ (table 2). No significant difference was observed in treatment regimens for nine patients with 5-year mortality. Among the patients, most of those with PPFE were treated with $\mathrm{RFP}+\mathrm{EB}+\mathrm{CAM}$-based regimens, except for one receiving an EB+CAM-based regimen, whereas, those without PPFE received an RFP+EB+CAM-based regimen. There were no significant differences in sex, percentage of smokers, previous histories of tuberculosis, presence of comorbidities, use of immunosuppressants, rate of positive sputum AFB smear, causative organisms, radiological MAC-PD type, MAC-PD status, and pulmonary symptoms, including cough, sputum, haemoptysis, or fever $(\mathrm{p}>0.05)$. After multidrug antimicrobial treatment, sputum AFB smear did not turn negative in 24 patients without PPFE and in 9 with PPFE $(\mathrm{p}>0.05)$. During or after the treatment, sputum AFB smear became positive again in nine patients with PPFE and without PPFE, respectively, without a significant statistical difference $(\mathrm{p}>0.05)$.

\section{Risk factor analysis for MAC-PD symptoms}

Although there was no significant difference in Fisher's exact test, multivariate logistic regression analysis showed that a higher PPFE score was a risk factor for dyspnoea (adjusted OR 1.36, 95\% CI 1.07-1.74, $\mathrm{p}=0.013$, supplementary table 3 ). Moreover, it was associated with the presence of fatigue and lower BMI (fatigue-adjusted OR 1.54, 95\% CI 1.11-2.14, $\mathrm{p}=0.010$, supplementary table 4; BMI-standardised partial regression coefficient $(\beta)=-0.229, \mathrm{p}<0.001$, table 3$)$. However, the PPFE score did not significantly affect the presence of cough, sputum, haemoptysis, or fever ( $p>0.05$, supplementary tables $5-8$ ).

On the MAC-PD scores, a higher consolidation score increased the risk of cough and fever (cough-adjusted OR 1.83, 95\% CI 1.09-3.08, p=0.022; fever-adjusted OR 2.24, 95\% CI 1.06-4.76, p=0.036). Higher cellular bronchiolitis and cavity scores were associated with haemoptysis (cellular 


\section{TABLE 1 Clinical characteristics (n=224)}

\begin{tabular}{|c|c|}
\hline Age years & $67.0 \pm 11.9(31-95)$ \\
\hline Male/female $\mathrm{n}$ & $50 / 174$ \\
\hline $\mathrm{BMI} \mathbf{k g} \cdot \mathrm{m}^{-2}$ & $19.3 \pm 2.6(13.2-26.8)$ \\
\hline Smoker & $64(28.6)$ \\
\hline Smoking pack-years & $9 \pm 20(0-135)$ \\
\hline Previous history of pulmonary TB & 28 (12.5) \\
\hline Comorbidities & $56(25.0)$ \\
\hline Asthma & $19(8.5)$ \\
\hline Diabetes mellitus & $15(6.7)$ \\
\hline COPD & $9(4.0)$ \\
\hline Rheumatoid arthritis & $7(3.1)$ \\
\hline Chronic interstitial pneumonia & $4(1.8)$ \\
\hline Chronic heart failure & $2(0.9)$ \\
\hline Pneumoconiosis & $1(0.4)$ \\
\hline Use of immunosuppressants & $8(3.6)$ \\
\hline Symptoms & $170(75.9)$ \\
\hline Cough & 141 (62.9) \\
\hline Sputum & $108(48.2)$ \\
\hline Dyspnoea & $38(17.0)$ \\
\hline Haemoptysis & 49 (21.9) \\
\hline Fever & 23 (10.3) \\
\hline Fatigue & $15(6.7)$ \\
\hline Weight loss & $47(21.0)$ \\
\hline Positive sputum AFB smear & $74(33.0)$ \\
\hline \multicolumn{2}{|l|}{ Causative organisms } \\
\hline Mycobacterium avium & $139(62.1)$ \\
\hline Mycobacterium intracellulare & $101(45.1)$ \\
\hline Co-infections $\#$ & $16(7.1)$ \\
\hline \multicolumn{2}{|l|}{ Radiological types } \\
\hline Nodular bronchiectatic & $173(77.2)$ \\
\hline Fibrocavitary & $48(21.4)$ \\
\hline Unclassifiable & $3(1.3)$ \\
\hline MAC-PD score & $9.0 \pm 4.6(2.0-26.0)$ \\
\hline \multicolumn{2}{|l|}{ MAC-PD status } \\
\hline Progressive disease & $103(46.0)$ \\
\hline Stationary disease ${ }^{+}$ & $121(54.0)$ \\
\hline PPFE score & $0.9 \pm 1.4(0.0-7.0)$ \\
\hline Limited & $56(25.0)$ \\
\hline Extensive & $32(14.3)$ \\
\hline Presence of PPFE & 59 (26.3) \\
\hline Presence of apical cap & $29(12.9)$ \\
\hline
\end{tabular}

Data are presented as mean \pm SD (range) or $n(\%)$, unless otherwise stated. BMI: body mass index; TB: tuberculosis; AFB: acid-fast bacilli; MAC-PD: M. avium complex pulmonary disease; PPFE: pleuroparenchymal fibroelastosis. " : co-infections of $M$. avium and $M$. intracellulare; ": a case requiring multidrug treatment within 3 years of diagnosis due to clinical or radiological deterioration; ${ }^{+}$: a case stable for $\geqslant 3$ years after diagnosis without clinical or radiological deterioration.

bronchiolitis-adjusted OR 1.30, 95\% CI 1.03-1.65, p=0.028; cavity-adjusted OR 1.20, 95\% CI 1.04-1.38, $\mathrm{p}=0.013)$. Although bronchiectasis and nodule scores correlated with cough and fever on univariate analysis $(\mathrm{p}<0.05)$, there was no significance on multivariate logistic regression analysis.

Among the clinical characteristics, only a positive sputum AFB smear, BMI, and causative organisms were significantly correlated with clinical symptoms of MAC-PD. A positive sputum AFB smear was associated with the presence of cough, sputum, and fever (cough-adjusted OR 2.27, 95\% CI 1.12-4.60, p=0.023; sputum-adjusted OR 2.56, 95\% CI 1.38-4.76, $\mathrm{p}=0.003$; fever-adjusted OR 3.39, 95\% CI 1.12-10.20, $\mathrm{p}=0.031$ ). A lower BMI was associated with an increased risk of fever (adjusted OR 0.78, 95\% CI $0.62-0.99, \mathrm{p}=0.042$ ), and $M$. avium infection was a factor for higher BMI (standardised partial $\beta=0.144$, $\mathrm{p}=0.026$ ). Other clinical characteristics, including smoking status, presence of comorbidities, previous history of pulmonary tuberculosis, and use of immunosuppressants did not correlate with any of the clinical symptoms $(\mathrm{p}>0.05)$. 


\begin{tabular}{|c|c|c|c|}
\hline Characteristic & $\begin{array}{l}\text { Patients with PPFE } \\
\text { (n=59) }\end{array}$ & $\begin{array}{l}\text { Patients without PPFE } \\
\text { (n=165) }\end{array}$ & p-value \\
\hline Age years & $70.3 \pm 11.6$ & $65.8 \pm 11.8$ & 0.013 \\
\hline Male/female $n$ & $10 / 49$ & $40 / 125$ & 0.279 \\
\hline $\mathrm{BMI} \mathrm{kg} \cdot \mathrm{m}^{-2}$ & $17.9 \pm 2.3$ & $19.8 \pm 2.6$ & $<0.001$ \\
\hline Smoker & $13(22.0)$ & $51(30.9)$ & 0.241 \\
\hline Smoking pack-years & $3.8 \pm 9.4$ & $10.2 \pm 22.2$ & 0.032 \\
\hline Previous history of pulmonary TB & 10 (16.9) & $18(10.9)$ & 0.254 \\
\hline Presence of comorbidities & $12(20.3)$ & $44(26.7)$ & 0.384 \\
\hline Use of immunosuppressants & $2(3.4)$ & $6(3.6)$ & $>0.999$ \\
\hline Symptoms & $46(78.0)$ & 124 (75.2) & 0.726 \\
\hline Cough & $40(67.8)$ & $101(61.2)$ & 0.433 \\
\hline Sputum & $31(52.5)$ & $77(46.7)$ & 0.452 \\
\hline Dyspnoea & $14(23.7)$ & $24(14.5)$ & 0.111 \\
\hline Haemoptysis & 18 (30.5) & 31 (18.8) & 0.069 \\
\hline Fever & 9 (15.3) & $14(8.5)$ & 0.209 \\
\hline Fatigue & $8(13.6)$ & $7(4.2)$ & 0.028 \\
\hline Weight loss & 20 (33.9) & $27(16.4)$ & 0.008 \\
\hline $\begin{array}{l}\text { Positive sputum AFB smear } \\
\text { Causative organisms } n\end{array}$ & 20 (33.9) & $54(32.7)$ & 0.873 \\
\hline Mycobacterium avium & 35 (59.3) & $104(63.0)$ & 0.641 \\
\hline Mycobacterium intracellulare & $26(44.1)$ & $75(45.5)$ & 0.880 \\
\hline Co-infections ${ }^{\#}$ & $2(3.4)$ & $14(8.5)$ & 0.249 \\
\hline \multicolumn{4}{|l|}{ Radiological types } \\
\hline Nodular bronchiectatic & 43 (72.9) & 130 (78.8) & 0.369 \\
\hline Fibrocavitary & $16(27.1)$ & $32(19.4)$ & 0.267 \\
\hline Unclassifiable & $0(0.0)$ & $3(1.8)$ & 0.568 \\
\hline MAC-PD score & $10.8 \pm 4.6$ & $8.4 \pm 4.4$ & $<0.001$ \\
\hline Bronchiectasis & $3.9 \pm 1.8$ & $2.7 \pm 1.8$ & $<0.001$ \\
\hline Cellular bronchiolitis & $3.7 \pm 1.4$ & $3.3 \pm 1.7$ & 0.084 \\
\hline Cavity & $1.6 \pm 2.6$ & $1.2 \pm 2.3$ & 0.270 \\
\hline Nodule & $0.6 \pm 0.6$ & $0.5 \pm 0.6$ & 0.609 \\
\hline Consolidation & $1.0 \pm 0.7$ & $0.6 \pm 0.7$ & 0.002 \\
\hline \multicolumn{4}{|l|}{ MAC-PD status } \\
\hline Progressive disease $e^{\pi}$ & 31 (52.5) & $72(43.6)$ & 0.287 \\
\hline Stationary disease ${ }^{+}$ & $28(47.5)$ & $93(56.4)$ & \\
\hline All-cause mortality within 5 years & 8 (13.6) & $1(0.6)$ & $<0.001$ \\
\hline
\end{tabular}

Data are presented as mean \pm SD or $n(\%)$, unless otherwise stated. BMI: body mass index; TB: tuberculosis; AFB: acid-fast bacilli; MAC-PD: M. avium complex pulmonary disease. " : co-infections of M. avium and M. intracellulare; ": a case requiring multidrug treatment within 3 years of diagnosis due to clinical or radiological deterioration; ${ }^{+}$: a case stable for $\geqslant 3$ years after diagnosis without clinical or radiological deterioration.

\section{Correlation analysis between PPFE and MAC-PD scores}

Both univariate and multivariate regression analyses found the PPFE score to correlate positively with the bronchiectasis score and negatively with the BMI (bronchiectasis score-standardised partial $\beta=0.260$, $\mathrm{p}<0.001$; BMI-standardised partial $\beta=-0.238, \mathrm{p}<0.001$, table 4). The consolidation score was associated with the PPFE score on univariate analysis (standardised $\beta=0.211, p=0.002$ ) but was not significantly associated on multivariate analysis $(\mathrm{p}=0.574)$. This indicates that patients with severe bronchiectasis are likely to have greater extent of PPFE.

\section{Risk factor analysis for progressive MAC-PD based on the presence of PPFE}

The PPFE score did not affect MAC-PD status significantly on univariate analysis ( $\mathrm{p}=0.576$, table 5). Although all MAC-PD scores, NB type, positive sputum AFB smear, and BMI correlated with MAC-PD progression on univariate analysis $(\mathrm{p}<0.05)$, only the consolidation score, positive sputum AFB smear, and older age were independent risk factors for progressive MAC-PD (consolidation score-adjusted OR 2.74, 95\% CI 1.54-4.85, $\mathrm{p}<0.001$; positive sputum AFB smear-adjusted OR 2.33, 95\% CI 1.11-4.89, $\mathrm{p}=0.026$; age-adjusted OR $0.95,95 \%$ CI $0.92-0.98, \mathrm{p}=0.001$ ). 


\begin{tabular}{|c|c|c|c|c|c|}
\hline \multirow[t]{2}{*}{ Characteristic } & \multicolumn{3}{|c|}{ Univariate analysis } & \multicolumn{2}{|c|}{ Multivariate analysis } \\
\hline & $\begin{array}{c}\text { Standardised } \\
\beta\end{array}$ & $\begin{array}{l}\text { Adjusted } \\
\mathbf{R}^{2}\end{array}$ & p-value & $\begin{array}{l}\text { Standardised } \\
\text { partial } \beta\end{array}$ & p-value \\
\hline PPFE score & -0.302 & 0.088 & $<0.001$ & -0.229 & $<0.001$ \\
\hline Bronchiectasis score & -0.236 & 0.052 & $<0.001$ & -0.047 & 0.559 \\
\hline Cellular bronchiolitis score & -0.050 & -0.002 & 0.478 & & \\
\hline Cavity score & -0.169 & 0.025 & 0.013 & -0.064 & 0.631 \\
\hline Nodule extent score & -0.077 & 0.001 & 0.271 & & \\
\hline Consolidation score & -0.170 & 0.025 & 0.014 & -0.016 & 0.827 \\
\hline $\begin{array}{l}\text { Radiological type (nodular } \\
\text { bronchiectatic) }\end{array}$ & 0.136 & 0.015 & 0.046 & 0.124 & 0.360 \\
\hline \multicolumn{6}{|l|}{ Causative organisms } \\
\hline Mycobacterium avium & 0.191 & 0.031 & 0.007 & 0.144 & 0.026 \\
\hline Mycobacterium intracellulare & -0.121 & 0.010 & 0.086 & & \\
\hline Positive sputum AFB smear & -0.048 & -0.003 & 0.492 & & \\
\hline Age & -0.204 & 0.038 & 0.003 & -0.182 & 0.007 \\
\hline Sex (female) & -0.223 & 0.046 & 0.001 & -0.263 & $<0.001$ \\
\hline Smoking pack-years & 0.128 & 0.012 & 0.065 & & \\
\hline Presence of comorbidities & 0.049 & -0.002 & 0.475 & & \\
\hline Previous history of pulmonary TB & -0.010 & -0.005 & 0.886 & & \\
\hline Use of immunosuppressants & -0.119 & 0.011 & 0.075 & & \\
\hline
\end{tabular}

Risk factor analysis for all-cause mortality within 5 years from MAC-PD diagnosis based on the presence of PPFE

Logistic regression analysis was conducted to determine factors associated with all-cause mortality within 5 years from the initial MAC-PD diagnosis (table 6). PPFE, bronchiectasis, cavity, and consolidation scores were associated with 5 -year mortality on univariate analysis (all $\mathrm{p}<0.05$ ). However, multivariate analysis found only a higher PPFE score and lower BMI to be significant and independent risk factors of 5-year

TABLE 4 Univariate and multivariate regression analyses for pleuroparenchymal fibroelastosis score $(n=224)$

\begin{tabular}{|c|c|c|c|c|c|}
\hline \multirow[t]{2}{*}{ Characteristic } & \multicolumn{3}{|c|}{ Univariate analysis } & \multicolumn{2}{|c|}{ Multivariate analysis } \\
\hline & $\begin{array}{c}\text { Standardised } \\
\beta\end{array}$ & $\begin{array}{l}\text { Adjusted } \\
\mathbf{R}^{2}\end{array}$ & p-value & $\begin{array}{l}\text { Standardised } \\
\text { partial } \beta\end{array}$ & p-value \\
\hline Bronchiectasis score & 0.329 & 0.104 & $<0.001$ & 0.260 & $<0.001$ \\
\hline Cellular bronchiolitis score & 0.058 & -0.001 & 0.391 & & \\
\hline Cavity score & -0.013 & -0.004 & 0.842 & & \\
\hline Nodule extent score & 0.015 & -0.004 & 0.821 & & \\
\hline Consolidation score & 0.211 & 0.040 & 0.002 & 0.041 & 0.574 \\
\hline $\begin{array}{l}\text { Radiological type (nodular } \\
\text { bronchiectatic) }\end{array}$ & 0.048 & -0.002 & 0.477 & & \\
\hline \multicolumn{6}{|l|}{ Causative organisms } \\
\hline Mycobacterium avium & -0.065 & 0.000 & 0.336 & & \\
\hline Mycobacterium intracellulare & 0.043 & -0.003 & 0.521 & & \\
\hline Positive sputum AFB smear & 0.043 & -0.003 & 0.518 & & \\
\hline Age & 0.151 & 0.018 & 0.024 & 0.009 & 0.904 \\
\hline Sex (female) & 0.078 & 0.002 & 0.245 & -0.012 & 0.875 \\
\hline BMI & -0.307 & 0.088 & $<0.001$ & -0.238 & $<0.001$ \\
\hline Smoking pack-years & -0.112 & 0.011 & 0.068 & & \\
\hline Presence of comorbidities & -0.037 & -0.003 & 0.578 & & \\
\hline Previous history of pulmonary TB & 0.069 & 0.000 & 0.306 & & \\
\hline Use of immunosuppressants & 0.007 & -0.004 & 0.923 & & \\
\hline
\end{tabular}




\begin{tabular}{|c|c|c|c|c|}
\hline \multirow[t]{2}{*}{ Characteristic } & \multicolumn{2}{|c|}{ Univariate analysis } & \multicolumn{2}{|c|}{ Multivariate analysis } \\
\hline & p-value & OR $(95 \% \mathrm{CI})$ & p-value & Adjusted OR $(95 \% \mathrm{CI})$ \\
\hline PPFE score & 0.576 & & & \\
\hline Bronchiectasis score & 0.008 & $1.22(1.05-1.41)$ & 0.805 & \\
\hline Cellular bronchiolitis score & 0.018 & $1.22(1.04-1.45)$ & 0.182 & \\
\hline Cavity score & $<0.001$ & $1.50(1.29-1.74)$ & 0.278 & \\
\hline Nodule extent score & 0.012 & $1.86(1.14-3.02)$ & 0.582 & \\
\hline Consolidation score & $<0.001$ & $2.98(1.94-4.60)$ & $<0.001$ & $2.74(1.54-4.85)$ \\
\hline Radiological type (nodular bronchiectatic) & $<0.001$ & $0.14(0.06-0.29)$ & 0.323 & \\
\hline \multicolumn{5}{|l|}{ Causative organisms } \\
\hline Mycobacterium avium & 0.981 & & & \\
\hline Mycobacterium intracellulare & 0.880 & & & \\
\hline Positive sputum AFB smear & $<0.001$ & $3.81(2.11-6.88)$ & 0.026 & $2.33(1.11-4.89)$ \\
\hline Age & 0.194 & & 0.001 & $0.95(0.92-0.98)$ \\
\hline Sex (female) & 0.518 & & 0.324 & \\
\hline BMI & 0.027 & $0.89(0.79-0.99)$ & 0.086 & \\
\hline Smoking pack-years & 0.525 & & & \\
\hline Presence of comorbidities & 0.190 & & & \\
\hline Previous history of pulmonary TB & 0.723 & & & \\
\hline Use of immunosuppressants & 0.817 & & & \\
\hline
\end{tabular}

mortality (PPFE score-adjusted OR 1.66, 95\% CI 1.06-2.60, variance inflation factor=1.47, $\mathrm{p}=0.028$; BMI-adjusted OR 0.61, 95\% CI 0.39-0.94, variance inflation factor=1.27, $\mathrm{p}=0.028$ ).

\section{Discussion}

This is the first comprehensive examination of the prevalence, clinical characteristics, and the prognostic impact of PPFE in patients with MAC-PD disease. This study highlights three major findings regarding PPFE in MAC-PD: 1) PPFE is a relatively common radiological characteristic and suggests a poor

TABLE 6 Risk factor analysis for all-cause mortality within 5 years from initial Mycobacterium avium pulmonary disease (MAC-PD) diagnosis ( $\mathrm{n}=224)$

\begin{tabular}{|c|c|c|c|c|}
\hline \multirow[t]{2}{*}{ Characteristic } & \multicolumn{2}{|c|}{ Univariate analysis } & \multicolumn{2}{|c|}{ Multivariate analysis } \\
\hline & p-value & OR $(95 \% \mathrm{CI})$ & p-value & Adjusted OR (95\% CI) \\
\hline PPFE score & $<0.001$ & $1.76(1.26-2.45)$ & 0.028 & $1.66(1.06-2.60)$ \\
\hline Bronchiectasis score & 0.005 & $1.57(1.15-2.15)$ & 0.626 & \\
\hline Cellular bronchiolitis score & 0.766 & & & \\
\hline Cavity score & 0.003 & $1.41(1.12-1.78)$ & 0.210 & \\
\hline Nodule extent score & 0.055 & & & \\
\hline Consolidation score & 0.005 & $3.40(1.45-7.99)$ & 0.733 & \\
\hline Radiological type (nodular bronchiectatic) & 0.027 & $0.22(0.06-0.84)$ & 0.763 & \\
\hline \multicolumn{5}{|l|}{ Causative organisms } \\
\hline Mycobacterium avium & 0.683 & & & \\
\hline Mycobacterium intracellulare & 0.968 & & & \\
\hline Positive sputum AFB smear & 0.157 & & & \\
\hline Age & 0.078 & & 0.498 & \\
\hline Sex (female) & 0.424 & & 0.062 & \\
\hline BMI & 0.002 & $0.57(0.40-0.81)$ & 0.028 & $0.61(0.39-0.94)$ \\
\hline Smoking pack-years & 0.717 & & & \\
\hline Presence of comorbidities & 0.182 & & & \\
\hline Previous history of pulmonary TB & 0.898 & & & \\
\hline Use of immunosuppressants & 0.992 & & & \\
\hline MAC-PD status (progressive disease) & 0.217 & & & \\
\hline
\end{tabular}


prognosis in patients with MAC-PD; 2) PPFE is a risk factor for low BMI and weight loss in patients with MAC-PD; and 3) PPFE becomes more extensive as bronchiectatic lesions progress in patients with MAC-PD

To date, some clinical characteristics predicting disease progression or poor prognosis have been reported in MAC-PD. A positive sputum AFB smear and older age reportedly predict a poor prognosis in patients with MAC-PD [20]. The results of the present study are similar with a positive sputum AFB smear and older age predicting MAC-PD progression. While $M$. intracellulare infection was not associated with treatment initiation within 3 years in the present study or the study by HwANG et al. [5], КоH et al. [20] reported that $M$. intracellulare infection was an independent factor for poor prognosis. Since patients with M. avium infection were more likely to maintain their BMI in this study (table 3), M. avium infection might be less invasive than $M$. intracellulare.

It is well known that the NB phenotype develops chronically while the FC phenotype develops rapidly $[4,22]$. Supporting this radiological classification, the presence of cavity and consolidation predict a rapid progression necessitating antimicrobial treatment [18]. Chronic interstitial pneumonia as a comorbidity is also reportedly an individual predictive factor necessitating antimicrobial treatment for MAC-PD [5]. However, no study has yet assessed the clinical association between PPFE and MAC-PD. Therefore, we investigated the prevalence, clinical characteristics, and prognostic impact of PPFE on MAC-PD.

The present study demonstrated that radiological PPFE is a relatively common poor prognostic factor in patients with MAC-PD. Previous studies indicated that BMI decreased when associated with MAC-PD in both the short and long term $[5,23]$. In this study, a lower BMI was associated with all-cause mortality within 5 years of the initial diagnosis. Although lower BMI predicted not only a poor prognosis but the radiological extent of PPFE, this study found that the extent of radiological PPFE was independently correlated with all-cause mortality within 5 years (tables 4 and 6). As patients with PPFE have decreased forced vital capacity and total lung capacity $[10,13]$ and have more frequent dyspnoea (supplementary table 3), subsequent restrictive ventilatory deficiency might result in a worse prognosis. Further studies to assess interactions between pulmonary function, BMI, and prognosis in patients with MAC-PD would be desirable.

PPFE accompanied with MAC-PD is a risk factor for lower BMI. Although weight loss and low BMI were reported in idiopathic PPFE and in the disease secondary to systemic sclerosis and idiopathic pulmonary fibrosis $[11,13,24]$, no study has yet reported on the clinical impact of PPFE on BMI in patients with PPFE associated with MAC-PD. Consistent with other studies of secondary PPFE, the present study demonstrated that patients with MAC-PD and PPFE had a lower BMI (tables 2 and 3). Patients with PPFE may develop platythorax as a result of marked upper lobe volume contraction [25] and they develop an increased residual volume/total lung capacity ratio as the disease progresses [26]. Consequently, patients with PPFE develop a restrictive ventilatory deficiency and require a stronger inspiratory drive to deliver sufficient inspiratory volume [26]. As forced vital capacity declines rapidly in patients with radiologically diagnosed PPFE [27], respiratory muscle fatigue due to platythorax and subsequent anorexia might have an impact on progressive weight loss and low BMI regardless of PPFE aetiology. This study provides a clinical foundation for further validating this hypothesis.

PPFE might progress as chronic bronchopulmonary inflammation due to MAC-PD increases. Recurrent bronchopulmonary infection commonly occurs as a comorbidity in patients with PPFE [10], and NTM infection frequently accompanies the disease course of PPFE [7]. However, there has been little investigation of the pathogenesis of PPFE accompanied with MAC-PD. The present study found that the extent of radiological PPFE correlated positively with bronchiectasis severity and extent in patients with MAC-PD (table 4). As traction bronchiectasis can occur during PPFE progression [28], the association between PPFE and bronchiectasis scores might merely suggest the presence of traction bronchiectasis. However, these results imply that both bronchiectasis and PPFE in MAC-PD might be attributable to the same mechanism. Given that bronchiectasis and cavity extent are associated with cytokine levels reflecting immune responses $[29,30]$, chronic or recurrent bronchopulmonary inflammation due to MAC infection might have affected both PPFE and bronchiectasis. As not receiving timely treatment is a risk factor for mortality in MAC-PD [31], early antimicrobial and/or anti-inflammatory treatment for MAC-PD might delay PPFE progression. However, the results of this study found that the presence of PPFE did not affect physicians' initiation of treatment for MAC-PD (table 5). Further studies to assess whether incorporating the presence of PPFE into a clinical risk index for initiating multidrug antimicrobial treatment for MAC-PD would be needed to better delineate this issue.

This retrospective study had some limitations. First, this was a single-centre retrospective study, and selection bias might have affected the findings. Second, this study was the first to investigate the presence of PPFE in patients with MAC-PD, and appropriate sample sizes were not calculated. Therefore, this study 
included only a small number of patients with all-cause mortality within 5 years of diagnosis. Further large-scale studies to validate the results of this study are necessary. Third, bronchiectatic lesions of MAC-PD predominant in the upper lobe can be difficult to be strictly differentiated from PPFE. However, the present study is still notable because it showed that upper lobe-dominant PPFE-like lesions might have an impact on the prognosis of MAC-PD. Further histological investigations to differentiate these findings should be conducted. Finally, this study did not perform spirometry, and the long-term impact of PPFE on pulmonary function in patients with MAC-PD should be investigated in further studies.

In conclusion, the present study assessed the prevalence, clinical characteristics, and prognostic impact of PPFE on MAC-PD. Radiological PPFE is a relatively common complication of MAC-PD. Patients with MAC-PD and PPFE have dyspnoea, fatigue, and weight loss more frequently than those without PPFE. Although the presence of radiological PPFE did not affect physicians' decisions on initiating multidrug antimicrobial treatment in this study, the presence of PPFE might predict a worse prognosis in patients with MAC-PD. Therefore, further studies to assess decision-making on the initiation of treatment seem to be warranted.

Author contributions: Conceptualisation and design: Y. Yamamoto; methodology: Y. Yamamoto; data collection Y. Yamamoto, K. Tsujino, T. Kuge, F. Okabe, T. Matsuki, T. Kawasaki, H. Kagawa; analysis and interpretation of data: M. Miki, K. Miki, M. Mori; writing the original draft: Y. Yamamoto; supervision: H. Kida. All of the authors reviewed and approved the submission of the final manuscript.

Availability of data and materials: All data generated or analysed during this study are included in this published article and its supplementary information files.

Conflict of interest: None declared.

\section{References}

1 Namkoong H, Kurashima A, Morimoto K, et al. Epidemiology of pulmonary nontuberculous mycobacterial disease, Japan. Emerg Infect Dis 2016; 22: 1116-1117.

2 Prevots DR, Marras TK. Epidemiology of human pulmonary infection with nontuberculous mycobacteria: a review. Clin Chest Med 2015; 36: 13-34.

3 Koh WJ, Moon SM, Kim SY, et al. Outcomes of Mycobacterium avium complex lung disease based on clinical phenotype. Eur Respir J 2017; 50: 1602503.

4 Griffith DE, Aksamit T, Brown-Elliott BA, et al. An official ATS/IDSA statement: diagnosis, treatment, and prevention of nontuberculous mycobacterial diseases. Am J Respir Crit Care Med 2007; 175: 367-416.

5 Hwang JA, Kim S, Jo KW, et al. Natural history of Mycobacterium avium complex lung disease in untreated patients with stable course. Eur Respir J 2017; 49: 1600537.

6 Fukushima K, Kitada S, Abe Y, et al. Long-term treatment outcome of progressive Mycobacterium avium complex pulmonary disease. J Clin Med 2020; 9: 1315.

7 Khiroya R, Macaluso C, Montero MA, et al. Pleuroparenchymal fibroelastosis: a review of histopathologic features and the relationship between histologic parameters and survival. Am J Surg Pathol 2017; 41: 1683-1689.

8 Amitani R, Niimi A, Kuse F. [Idiopathic pulmonary upper lobe fibrosis]. Kokyu 1992; 11: 693-699.

9 Frankel SK, Cool CD, Lynch DA, et al. Idiopathic pleuroparenchymal fibroelastosis. Chest 2004; 126: $2007-2013$.

10 Reddy TL, Tominaga M, Hansell DM, et al. Pleuroparenchymal fibroelastosis: a spectrum of histopathological and imaging phenotypes. Eur Respir J 2012; 40: 377-385.

11 Bonifazi M, Sverzellati N, Negri E, et al. Pleuroparenchymal fibroelastosis in systemic sclerosis: prevalence and prognostic impact. Eur Respir J 2020; 56: 1902135.

12 Kurosaki F, Bando M, Nakayama M, et al. Clinical features of pulmonary aspergillosis associated with interstitial pneumonia. Intern Med 2014; 53: 1299-1306.

13 Oda T, Ogura T, Kitamura H, et al. Distinct characteristics of pleuroparenchymal fibroelastosis with usual interstitial pneumonia compared with idiopathic pulmonary fibrosis. Chest 2014; 146: 1248-1255.

14 Daley CL, Iaccarino JM, Lange C, et al. Treatment of nontuberculous mycobacterial pulmonary disease: an official ATS/ERS/ESCMID/IDSA clinical practice guideline: executive summary. Clin Infect Dis 2020; 71: e31-e36.

15 Jacob J, Odink A, Brun AL, et al. Functional associations of pleuroparenchymal fibroelastosis and emphysema with hypersensitivity pneumonitis. Respir Med 2018; 138: 95-101.

16 McLoud T, Isler R, Novelline R, et al. The apical cap. AJR Am J Roentgenol 1981; 137: 299-306.

17 Chua F, Desai SR, Nicholson AG, et al. Pleuroparenchymal fibroelastosis. a review of clinical, radiological, and pathological characteristics. Ann Am Thorac Soc 2019; 16: 1351-1359.

18 Lee G, Lee KS, Moon JW, et al. Nodular bronchiectatic Mycobacterium avium complex pulmonary disease Natural course on serial computed tomographic scans. Ann Am Throc Soc 2013; 10: 299-306.

19 Lee G, Kim HS, Lee KS, et al. Serial CT findings of nodular bronchiectatic Mycobacterium avium complex pulmonary disease with antibiotic treatment. AJR Am J Roentgenol 2013; 201: 764-772.

20 Koh WJ, Kwon OJ, Jeon K, et al. Clinical significance of nontuberculous mycobacteria isolated from respiratory specimens in Korea. Chest 2006; 129: 341-348.

21 Kanda Y. Investigation of the freely available easy-to-use software "EZR" for medical statistics. Bone Marrow Transplant 2013; 48: 452-458.

22 Reich JM, Johnson RE. Mycobacterium avium complex pulmonary disease presenting as an isolated lingular or middle lobe pattern. Chest 1992; 101: 1605-1609.

23 Yamazaki Y, Kubo K, Takamizawa A, et al. markers indicating deterioration of pulmonary Mycobacterium avium-intracellulare infection. Am J Respir Crit Care Med 1999; 160: 1851-1855. 
24 Enomoto Y, Nakamura Y, Satake Y, et al. Clinical diagnosis of idiopathic pleuroparenchymal fibroelastosis: a retrospective multicenter study. Respir Med 2017; 133: 1-5.

25 Harada T, Yoshida Y, Kitasato Y, et al. The thoracic cage becomes flattened in the progression of pleuroparenchymal fibroelastosis. Eur Respir Rev 2014; 23: 263-266.

26 Watanabe S, Waseda Y, Takato $\mathrm{H}$, et al. Pleuroparenchymal fibroelastosis: distinct pulmonary physiological features in nine patients. Respir Investig 2015; 53: 149-155.

27 Shioya M, Otsuka M, Yamada G, et al. Poorer prognosis of idiopathic pleuroparenchymal fibroelastosis compared with idiopathic pulmonary fibrosis in advanced stage. Can Respir J 2018; 2018: 1-7.

28 Namba M, Masuda T, Takao S, et al. Extent of pulmonary fibrosis on high-resolution computed tomography is a prognostic factor in patients with pleuroparenchymal fibroelastosis. Respir Investig 2020; in press [https://doi.org/ 10.1016/j.resinv.2020.05.009].

29 Vankayalapati R, Wizel B, Samten B, et al. Cytokine profiles in immunocompetent persons infected with Mycobacterium avium complex. J Infect Dis 2001; 183: 478-484.

30 Bamba $\mathrm{Y}$, Moro H, Aoki N, et al. Multiplex cytokine analysis in Mycobacterium avium complex lung disease: relationship between CXCL10 and poor prognostic factors. BMC Infect Dis 2019; 19: 263.

31 Wang PH, Pan SW, Shu CC, et al. Clinical course and risk factors of mortality in Mycobacterium avium complex lung disease without initial treatment. Respir Med 2020; 171: 106070 